ISSN: 2011-8643 | Vol. 2 | No. 4 | 2008 | pp. 63-79

\title{
Una propuesta epistemológica para el desarrollo de competencias en el SENA, desde el modelo constructivista
}

\author{
Blanca Aurora Pita Torres ${ }^{1}$ \\ Dora Ligia Páez Luna²
}

\section{Resumen}

En desarrollo de la investigación se analiza el enfoque de competencias adoptado por el SENA y su implementación a través del proceso de enseñanza-aprendizaje; trata de manera analítica la concepción de competencia y la interpretación que el SENA asume desde lo operacional, relegando los desarrollos cognitivos y formativos que dicho concepto

1 Licenciada en Filosofía e Historia de la Universidad Santo Tomás, Diplomada en Teología Espiritual de la Universidad Gregoriana de Roma, Diplomada en Archivistica y Diplomacia de la Escuela Vaticana de Roma, Diplomada en Investigación Cualitativa y Cuantitativa de la Universidad Santo Tomás, Magíster en Educación de la Universidad de la Sabana. Ha sido Rectora de Instituciones Educativas de Educación Básica y Media. En la Universidad Santo Tomás, Coordinadora de la Línea de Investigación de Currículo, Directora del Sistema de Investigación Educativa y Pedagógica, SIEP, de la Facultad de Educación. Actualmente Docente y Directora de la Maestría en Educación, desde donde lidera el grupo "Investigación y Docencia", reconocido por Colciencias y clasificado en Categoría B. Escribió la fundamentación conceptual del Área de Currículo, que se encuentra en los Portafolios I, II, III de la Maestría en Educación de la USTA. Dirección de contacto: blancaaurorapita@ustadistancia.edu.co.

2 Magíster en Educación, Universidad Santo Tomás Evaluadora y Auditora de Competencias Laborales del SENA. Metodóloga para la Elaboración de Normas de Competencia Laboral del SENA. Formadora Pedagógica Especializada en Formación Profesional con base en Competencias Laborales, del SENA. 
connota; la polarización se genera en el énfasis que la institución le otorga a la satisfacción de las demandas del sector productivo del país, desde su oferta educativa.

De este análisis se desprende una propuesta para la construcción de un marco epistemológico, cuya pretensión es la de fundamentar desde lo formativo el proceso de enseñanza-aprendizaje para el desarrollo de competencias, que en la actualidad adelanta el SENA. La propuesta busca subsanar las contradicciones entre el planteamiento del enfoque, el modelo pedagógico constructivista que desde lo teórico, sustenta su desarrollo y la acción real del docente en el aula de clase, la cual está sujeta al cumplimiento de Los Procedimientos para la Ejecución de Acciones de Formación Profesional, adoptados por la entidad y estandarizados por el sistema de gestión de calidad.

\section{Palabras clave}

Epistemología, modelo pedagógico, competencia, competencia laboral, hermenéutica, aprendizaje, enseñanza.

\section{Abstract}

This research analyzes the approach to competences SENA has adopted and its development throughout the teaching - learning process; in an analytical way, it deals with the conception of competence and the interpretation SENA assumes for its development from an operational point of view, pushing the cognitive and formative developments this concept implies into the background; this polarization results from the emphasis the institution grants to satisfying the demand of the country's productive sector, starting from its educational offer.

This analysis result in a proposal for creating an epistemological framework that intends to support from an educational perspective, the teaching - learning process for developing competences, SENA is getting on with at present, with a view to rectify the contradictions among the approach, the constructivist pedagogical model that supports its development and the teacher's actual action in the classroom, which is in compliance with the procedures for performing the integral education actions adopted by the entity and standardized by the Quality Management System.

\section{Key Words}

Epistemology, pedagogical model, competence, labor competences, hermeneutics, learning, teaching.

\section{Introducción}

En la actualidad el Servicio Nacional de Aprendizaje SENA desarrolla el Proceso de Formación Profesional Integral con el Enfoque de Competencias, el cual presenta grandes fortalezas, así como algunas debilidades de tipo epistemológico, que se hace necesario abordar a partir de, en primera instancia, una descripción de éstas y, en segundo lugar, con el diseño de una propuesta sobre cómo podrían ser subsanadas y transformadas en aspectos positivos.

El funcionalismo aplicado como filosofía del Sistema de Competencia Laboral en Inglaterra es la base sobre la cual el SENA edifica el enfoque de competencias. A éste le interesa la descripción del puesto o la función desempeñada por un trabajador; centra su atención en el resultado y no en el proceso que lo sustenta. 
En el campo de lo educativo, se evidencia la influencia del funcionalismo en el establecimiento de una serie de tareas que deben realizar tanto estudiante como docente; tareas que constituyen en sí mismas los pasos para concretar el proceso de enseñanzaaprendizaje; se convierten en el conjunto de acciones que evidencian quéy cómo se enseña, es decir, son la didáctica aplicada en el SENA.

A la serie de tareas mencionadas, el SENA las denomina: El Procedimiento para la Ejecución de Acciones de Formación Profesional Integral; al aplicarlo como delineador de los actos de enseñar y de aprender dificulta la generación de procesos creativos, autónomos, autogestionados. El procedimiento se presenta de manera rigurosa y secuencial; se soporta en el diligenciamiento de diversos formatos institucionales, sujetos a su vez, a las prescripciones establecidas por un sistema de gestión de calidad en la que están empeñados en la actualidad los centros de formación.

El carácter administrativo tanto de los procedimientos mencionados como el Sistema de Gestión de Calidad, enmarcan la didáctica propuesta para el desarrollo del enfoque; los dos componentes le otorgan mayor énfasis conductista a dicha acción, la cual se basa en la ejecuciones precisas y el control de las mismas, mediante instrumentos (listas de chequeo) que documentan el registro de conductas observables (desempeños), homologadas con el desarrollo intelectual. Esta concepción de aprendizaje prioriza la dimensión biofísica de la persona al centrarse en la verificación de habilidades y destrezas.

Los conocimientos son objeto de comprobación mediante cuestionarios, su diseño está prescrito en el Manual de Diseño Curricular para el Desarrollo de competencias en la Formación Profesional Integral; en la acción práctica del proceso de enseñanzaaprendizaje se relegan a un segundo lugar, para atender la prioridad institucional, la recolección de evidencias de desempeño y de producto.

La mayor preocupación de la didáctica institucional gira en torno al desarrollo de habilidades operativo-instrumentales, hecho que se identifica en gran medida con la Tecnología Educativa, basada en la propuesta realizada en los años 50 por el Psicólogo norteamericano Frederic Burrhos Skinner, conocida como: Instrucción Programada; la fijación de objetivos terminales y su logro mediante la instrucción son esenciales para garantizar el aprendizaje.

La característica que con mayor proximidad permite identificar la didáctica actual del enfoque de competencias con la instrucción programada, se encuentra en el agudo énfasis que se le otorga al resultado del proceso de enseñanza-aprendizaje, mediante el entrenamiento. Prevalecen los productos tangibles, cuantificables, verificables, que impacten la transformación de la realidad, especialmente, en el ámbito de lo técnico-productivo, relegando a un segundo lugar, la generación de procesos cognitivos, comunicativos, axiológicos; estos son, generalmente, de carácter intangible, escasamente medibles o verificables al menos, no en los mismos términos propuestos; pero, definitivamente constitutivos esenciales inherentes al aprendizaje y, con éste, a la competencia.

El énfasis en los desempeños y productos medibles, obtenidos mediante entrenamiento son las características propias del Enfoque para el Desarrollo de Competencias propuesto por el SENA dan forma a un problema de carácter epistemológico, dado que se propone desarrollar competencias en el estudiante, es decir altos niveles cognitivos, un sólido marco axiológico, alto nivel de tipo comunicativo mediante una metodología de carácter conductista, que apunta a la competencia eminentemente de carácter operativo, evidenciando así una sentida contradicción.

Esta contradicción es la que se aborda desde la presente investigación, a manera de propuesta para construir un marco epistemológico, cuyo propósito consiste en tratar de dar coherencia al enfoque de competencias del SENA con el modelo pedagógico constructivista, como una alternativa para la acción con sentido del estudiante. 
El modelo constructivista posibilita al estudiante, la apropiación personal de su proceso de aprendizaje, mediante escenarios de actividad significativa, en los que se evidencien todas y cada una de sus dimensiones personales, entre las que se encuentran como las más destacadas, la cognitiva, la axiológica, la comunicativa, la interpersonal, la motriz. Este tipo de didáctica es una oportunidad para que el estudiante ponga en escena todas y cada una de sus capacidades, en función de lograr el propósito clave del proceso de aprendizaje, cuál es el desarrollo humano y, cómo parte de éste, las competencias para el mundo de la vida.

La investigación se nutre con el diseño metodológico, estructurado desde el análisis de las experiencias y concepciones de los docentes del Centro de Gestión Administrativa del SENA - Regional Distrito Capital; así, como de la experiencia personal como Formadora de Docentes de la autora del presente documento; de las observaciones y acompañamientos realizados a los Docentes en su aula de clase y, del trabajo con empresarios e instituciones educativas en general.

La investigación presenta una propuesta para la construcción de un marco epistemológico que, desde el modelo pedagógico constructivista, fundamente el proceso de enseñanza-aprendizaje para el desarrollo de competencias, que imparte en la actualidad, el Servicio Nacional de Aprendizaje -SENA-; la propuesta se fundamenta en las interrelaciones generadas por los siguientes ejes temáticos: el epistemológico, el modelo pedagógico constructivista y el enfoque de competencias, a partir del tratamiento hermenéutico del concepto competencia.

\section{Antecedentes de la Investigación}

\section{Empíricos}

La política metodológica para el desarrollo de las acciones de formación profesional integral que imparte el SENA ha transcurrido históricamente a través de diferentes modelos y enfoques pedagógicos, hasta llegar a la actual propuesta del Enfoque para el Desarrollo de Competencias.

La evolución metodológica institucional connota una gestión formativa sistemática, los procesos formativos giran en torno a un método específico, con un propósito clave: la Formación Técnica para el Trabajo; el punto de inicio en la historia formativa del SENA, se concreta en los distintos centros de formación, las aulas de clase son escenarios fabriles, en donde se enseña, haciendo.

La consideración del aula de clase como taller de producción, es transformada gracias a los efectos, que en la formación profesional integral que imparte el SENA, tienen los acelerados y permanentes avances tecnológicos, la dinámica laboral, las renovaciones educativas, el desarrollo de los medios masivos de comunicación, sumado a la creciente demanda, se convierten en factores clave que impulsan a la institución a pensar en otro tipo de métodos de formación.

Son numerosas las alternativas metodológicas planteadas por el SENA, en el transcurso de sus cincuenta años de existencia, como respuesta a la dinámica de los factores antes mencionados. Metodologías que inician en el aula-taller, pasan por la autoformación, el aprendizaje por procesos, el aprender a aprender hasta llegar al actual enfoque para desarrollar competencias.

La característica común en cada una de las metodologías formativas desarrolladas por el SENA, consiste en la articulación de lo educativo con lo productivo, para ello, la formación, actualización y capacitación permanente se asume en consonancia con las necesidades del sector productivo del país.

Apuntar a satisfacer las necesidades del sector productivo implica, de una parte, el diseño de estructuras curriculares centradas en el perfil de un trabajador determinado con base en el estudio integral del trabajo; de otra, el desarrollo curri- 
cular sujeto a la acción, para lo cual se propone la didáctica centrada en el aprender a hacer, su complemento lo constituye, el aprender a aprender y el aprender a ser.

El aprender a aprender se orienta al desarrollo del aprendizaje por procesos y a la formación permanente; el aprender a ser, se orienta al desarrollo de actitudes y de las interrelaciones de la persona consigo mismo, con los demás y con la naturaleza.

A pesar de que en la literatura institucional se propende por los dos aprenderes como parte constitutiva esencial de la formación profesional integral; en la acción real institucional relacionada con el diseño y el desarrollo curricular, se omiten los módulos básicos o de política institucional en los que se fundamenta el desarrollo de las competencias básicas; es decir, el fortalecimiento de las dimensiones humanas relacionadas con el ser: valores, comunicaciones y altos niveles de pensamiento; éstas son la base sobre la cual se consolida el desarrollo humano, eje central de lo formativo.

Los módulos formativos que plasman los contenidos temáticos pertinentes con la dimensión humana, el ser, se ven reducidos a exiguas intensidades horarias, según el parecer de los innumerables diseñadores, hecho que de partida desmiembra la unidad pedagógica y técnica institucional, reduciendo al mínimo las condiciones requeridas para el desarrollo de las competencias básicas sobre las cuales se fundamentan las demás competencias (específicas, transversales); la interrelación sistémica de estos tres tipos de competencias dan sentido al concepto de educar.

La educación cobra significado cuando se aprende desde la vida y para la vida, desde el sentimiento y el afecto, desde la comunicación con el otro, desde la capacidad de discernimiento sobre lo bueno y lo malo; la razón, la lógica, la intelectualidad, la habilidad carecen de sentido si surgen de manera aislada, fría, medida y controlada por estándares, centrados en el "hacer" del proceso de aprendizaje, eje sobre el cual el SENA ha centrado su atención, casi de manera exclusiva, como garantía del desarrollo de competencias.

La propuesta formativa centrada en el desarrollo de competencias, surge en los años noventa como la alternativa de mayor pertinencia para acercar a los medios educativo y productivo; se basa en el análisis funcional adelantado en Inglaterra, que como ya se mencionó, consiste en determinar las funciones que cumple un trabajador para lograr un resultado satisfactorio en términos de productividad.

El estudio de las funciones, se concreta en las normas de competencia laboral, éstas son la base para el nuevo diseño curricular; su desarrollo está sujeto al seguimiento, por parte del docente del Procedimiento para la Ejecución de Acciones de Formación Profesional Integral y el cumplimiento de los procesos del Sistema de Gestión de Calidad establecidos para tal fin

\section{Bibliográficos}

Algunos de los principales estudios institucionales que se han realizado al respecto han sido de carácter histórico, a manera de monografías, entre los cuales se destacan 20 Años del SENA en Colombia 1957 - 1977, se presenta en conmemoración del vigésimo aniversario institucional; describe, las etapas fundamentales del desarrollo del SENA durante el lapso mencionado.

El segundo documento es SENA 45 años 1957 -2002, recuenta la historia de los 45 años del SENA retomando algunos aspectos fundamentales de la política educativa en formación para el trabajo, con énfasis en los procesos de reestructuración industrial y la informatización de sectores de la industria, el comercio, la agroindustria y los servicios, con ellos se transforman los perfiles ocupacionales, se determinan así, nuevas demandas de formación profesional.

Otros estudios realizados en la década de los ochenta: La Formación Permanente, Individualiza- 
da y Modulary La Formación del Futuro, presentan los principios fundamentales de la Formación Profesional según las tendencias, características y acuerdos alcanzados por instituciones de formación profesional de otros países como: El Servicio Nacional de Aprendizaje Industrial (SENAI) y el Servicio Nacional de Aprendizaje Comercial (SENAC) del Brasil, El Instituto Nacional de Aprendizaje (INA) de Costa Rica, El Instituto Nacional de Capacitación Empresarial (INCE) de Venezuela, con la Coordinación del Centro Interamericano para la Formación Profesional (CINTERFOR).

Además de presentar los principios de la Formación Profesional, el SENA busca la consolidación de la unidad técnica institucional mediante algunas metodologías que apuntan a reestructurar el sistema de formación-producción, a partir de la directriz: Orientaciones para los Procesos de Formación-Producción en los Centros; algunos de los manuales en este marco son: Manual de Enseñanza-Aprendizaje para la Modalidad Formación en Centros y Estrategias Didácticas para la Formación Profesional.

EI SENA en el marco del actual enfoque de competencias, ha complementado sus propuestas metodológicas con el estudio realizado sobre las competencias básicas, titulado: Estado del Arte de la Competencias Básicas o Esenciales; ha publicado manuales como: Manual de Diseño Curricular para el Desarrollo de Competencias en la Formación Profesional Integral y Manual para la Selección, Prescripción, Elaboración y Contratación de Medios de Comunicación Didácticos; los propósitos son presentar el conjunto de procedimientos y actividades tanto para diseñar estructuras curriculares -módulos de formaciónpara el desarrollo de competencias, como los relacionados con los medios didácticos.

Con el fin de unificar la diversidad del quehacer docente, orientar la acciones de Formación Profesional Integral y mejorar el nivel de comprensión frente al nuevo enfoque, el SENA propone como referente teórico: El Procedimiento para la Ejecución de Acciones de Formación Profesional Integral y la Guía Metodológica para la Administración de la Formación Profesional Integral del SENA.

Los estudios adelantados y las propuestas institucionales, especialmente las presentadas en el marco del enfoque, se orientan a discriminar mediante tareas soportadas en formatos la ejecución de las acciones del docente en la orientación y evaluación de los procesos educativos, constriñendo la autonomía y autogestión necesarias del proceso de aprendizaje.

Al fijar tareas precisas al docente, se posiciona el acto de enseñar sobre el ejercicio de aprender, se enfatiza la importancia de la acción del docente y de manera proporcional se minimiza el accionar del estudiante sobre su personal proceso de construcción de conocimiento y aunque contemplan, teóricamente, el desarrollo del ser y del saber como elementos fundamentales en el proceso de aprendizaje, el énfasis, en la práctica del aula, se encuentra en el hacer, es decir, en la actuación del estudiante basada en el desarrollo operativo de habilidades y destrezas.

En estas publicaciones no se encuentra claridad o precisión sobre qué significa conocer, en el proceso de enseñanza-aprendizaje; la relación entre el hacer y el saber adolece de una teoría que permita establecer la necesaria interdependencia entre estas dos dimensiones del aprendizaje, así como entender su natural convergencia en el desarrollo de la competencia. Razón por la cual, se hace necesario contar con una propuesta de carácter epistemológico, que supla el vacío teórico en el enfoque institucional.

\section{Diseño Metodológico}

Es una investigación cualitativa de carácter hermenéutico, basada en el análisis de la información obtenida desde los diversos referentes que constituyen el enfoque de competencias en el SENA - Regional Distrito Capital, específicamente, el relacionado con la práctica educativa de los docentes en el Centro de Gestión Administrativa. 
La acción práctica del docente es el acontecimiento en donde con mayor fuerza se hace evidente la contradicción epistemológica, objeto de investigación. Ésta se constituye en un hecho determinante para profundizar en la indagación y la focalización del problema planteado. Para registrar dicha práctica se aplicó una encuesta con el fin de obtener información primaria, se formularon preguntas cerradas y abiertas; éstas últimas, se incluyen con el fin de posibilitar la libre expresión de las experiencias y conceptos del docente frente al enfoque de competencias y el proceso formativo.

La encuesta es un instrumento valioso en el marco de la presente investigación, pues posibilita la recolección de información primaria de un grupo representativo de docentes, para el caso, los docentes del Centro de Gestión Administrativa del SENA - Regional Distrito Capital; dichos resultados se proyectaron al proceso formativo en el SENA, dado que tanto la política, como los procesos emanados por la Dirección General son los regentes de la gestión institucional.

Las líneas política y procedimental mencionadas buscan la estandarización a nivel nacional; de tal manera, que el acto educativo real, se da en muy similares condiciones en todos y cada uno de los Centros de Formación con los que cuenta el SENA en el país; la homogenización dada se constituye en factor determinante para interpretar y explicar, a partir de los hechos de un Centro, el Proceso de Enseñanza Aprendizaje en el Enfoque de Competencias de la institución.

El análisis de la información busca comprender la problemática, se basa en la práctica docente y pretende, ir más allá de ella; se propone inferir y establecer las interrelaciones entre el proceso de enseñanza-aprendizaje desde el Modelo Constructivista y el Enfoque de Competencias adoptado por el SENA, así como ser uno de los referentes para diseñar una propuesta que subsane las contradicciones que se presentan en sus interrelaciones.
La acción hermenéutica se nutre de la experiencia personal, como Formadora de Docentes, de las observaciones y acompañamientos realizados a los Docentes en el aula, así como del trabajo con empresarios e instituciones educativas.

El estudio aporta nuevas visiones a la formación profesional integral, al desarrollo de competencias, tanto al interior del SENA como a las instituciones educativas del país, especialmente aquellas que atienden la formación para el trabajo, al igual que universidades y colegios que se encuentran en proceso de integración con los programas y la metodología del SENA.

El método propuesto para abordar el presente objeto de investigación se constituye en una alternativa susceptible de ser aplicado en la ampliación, profundización y generación de nuevas investigaciones en el campo educativo. Posibilita dar cuenta de los hechos formativos, establecer relaciones, encontrar los principios de determinación que le son propios, fortalecer los procesos investigativos que requieren atención.

La población la constituye 190 docentes del SENA -Centro de Gestión Administrativa- Regional Distrito Capital, quienes en la actualidad orientan las estructuras curriculares de Asistencia Administrativa, Gestión del Talento Humano y Gestión Documental, según los lineamientos determinados por enfoque de competencias y lo establecido en el diseño curricular desde la norma de competencia laboral.

La muestra comprende 54 docentes que en la actualidad orientan los módulos específicos y transversales, que conforman cada una de las tres estructuras curriculares mencionadas; son docentes de planta y de contrato, vinculados estos últimos al SENA, en el segundo semestre del 2006 y en el año 2007.

La muestra fue tomada teniendo en cuenta la vinculación laboral de los docentes; en el caso de los docentes de planta, el menor rango de tiempo de vinculación con el SENA oscila entre diez (10) a 
quince (15) años, son profesionales con alto nivel de capacitación pedagógica brindada por la institución, y fortalecida en el medio externo mediante convenios con universidades nacionales.

Los docentes de contrato, que en la actualidad se encuentran en la institución, no requieren acreditar ningún tipo de perfil pedagógico para su contratación trimestral; este alto nivel de rotación genera, escaso conocimiento institucional, específicamente en lo relacionado con los procesos de la Formación Profesional Integral.

El tiempo de vinculación y el nivel de capacitación constituyen aspectos de fundamental significación para los resultados generales de la investigación. En el caso de los docentes de planta, el tiempo de permanencia con el SENA, los hace actores dinámicos de sus procesos; especialmente en el relacionado con las diversas metodologías de formación aplicadas a través de la historia institucional, entre las que se encuentra el desarrollo de competencias.

En cuanto a los docentes de contrato, al carecer de perfil pedagógico su acción formativa se rige por el sentido común y la experiencia como docentes en otras instituciones, condiciones representativas en el marco de la investigación planteada, dado su desconocimiento de las metodologías de formación específicamente, la relacionada con el enfoque de competencias.

\section{Resultados y Discusión}

Para conocer sobre el modelo constructivista como base para desarrollar el proceso de aprendizaje-enseñanza y el enfoque de competencias adoptado por el SENA, se consultó a los docentes mencionados, de cuyas respuestas se colige que:
- Entienden como conocimiento la acción mediante la cual, se transforma la realidad personal, social y laboral; que este proceso se genera en el escenario del aprendizaje a través de estrategias didácticas activas que impliquen actividad significativa permanente del estudiante, tanto en lo cognitivo como en lo operativo.

- La expresión: actividad significativa es asumida por el docente como la acción práctica que conduce a obtener resultados en términos de productos y procesos, preferiblemente en términos tangibles que apunten al mundo del trabajo; hecho que se evidencia cuando al cuestionársele sobre los marcos teóricos que sustentan el aprendizaje, los considera como: "nada importantes".

- Cuando se le solicita que mencionen los productos o procesos que desde su módulo de formación han aportado al desarrollo tecnológico y científico del país, el mayor rango de respuestas está en el ítem, "sin información"; lo cual deja expreso el hecho de que si los docentes no obtienen un producto concreto, un proceso tangible en el desarrollo de los módulos, consideran que no existe ningún aporte, y mucho menos el desarrollo de una competencia.

Lo anterior evidencia, que el énfasis en el resultado del proceso de enseñanza-aprendizaje como requisito indispensable en el desarrollo de la competencia, según el enfoque del SENA, ha logrado permear en el docente, conceptos sobre: qué significa aprender, qué significa enseñar, qué significa el desarrollo de la competencia, fortaleciendo la tendencia hacia considerar que lo concreto, lo demostrable (desempeños, productos), son la evidencia contundente de aprendizaje y, por lo tanto, de competencia. 
Señale el aporte que considera más significativo desde el desarrollo de los módulos que orienta en la actualidad: (Estructuras curriculares: Gestión del Talento Humano; Asistencia Administrativa, Gestión Documental)

1. Desarrollo personal y laboral

2. Mejoramiento de los procesos de la organización

3. Desarrollo tecnológico

4. Organización de la información.

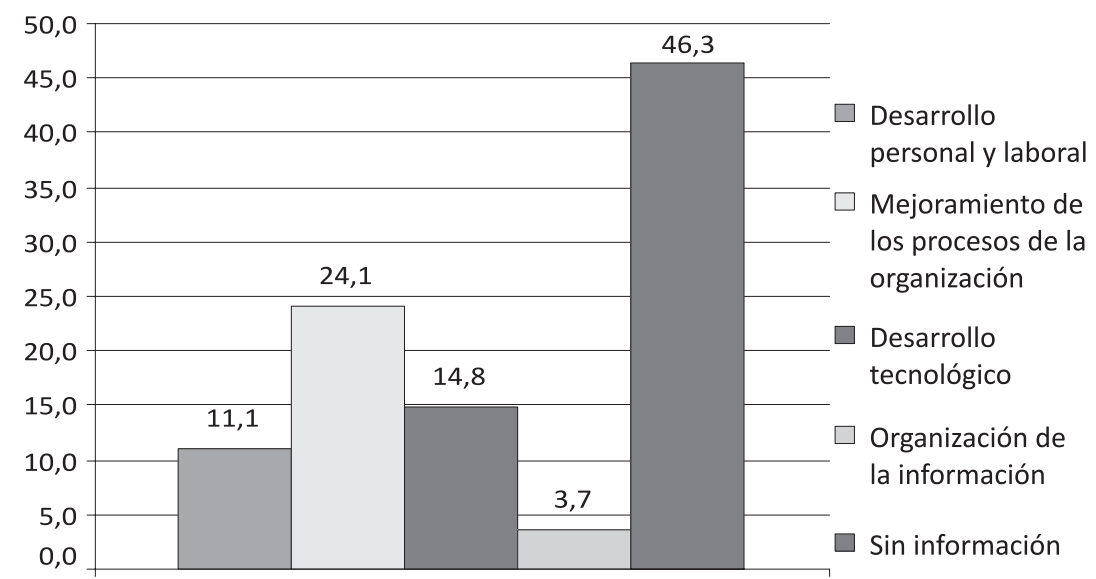

En cuanto a la interpretación que el docente hace sobre desarrollo de procesos de conocimiento, expresa: "hay procesos de desarrollo de conocimiento en el estudiante, cuando demuestra capacidad para transformar su realidad personal, social y laboral como consecuencia del nuevo conocimiento"; sin embargo, si esta transformación (una teoría, una nueva concepción de realidad) no es evidenciable de manera tangible, medible, cuantificable, no es considerada como aporte del proceso de aprendizaje.

En cuanto al desarrollo de conocimientos, el docente considera fundamental la metodología que aplique en el proceso de enseñanza-aprendizaje. Al respecto, se formuló la siguiente pregunta:

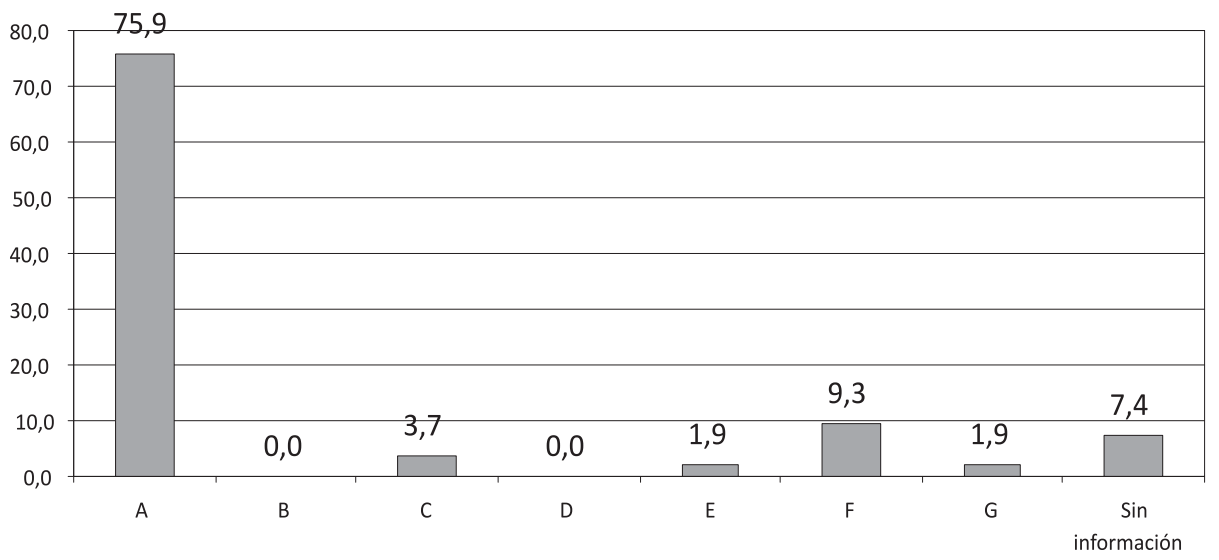


¿De qué forma, considera que es posible desarrollar conocimiento en el estudiante?

A. Presentando propuestas de estrategias de aprendizaje, que le impliquen al estudiante actividad significativa permanente, tanto en lo cognoscitivo como en lo operativo.

B. Es un proceso propio y personal del estudiante ante el cual, el docente no tiene mayor influencia.

C. Diseñando guías de enseñanza-aprendizaje que correspondan al desarrollo de las temáticas del módulo de formación.

D. Dependen de manera exclusiva de los niveles de motivación del estudiante frente a su proceso de aprendizaje por lo tanto, el Docente no tiene ninguna influencia.

E. Evaluando permanentemente el proceso de enseñanza-aprendizaje.

F. Solicitando resultados, cuya evidencia se da mediante desempeños $\mathrm{y} / \mathrm{o}$ productos.

G. Todas las anteriores

Sin información

El mayor peso de respuesta se da en la opción $A$, con un $75.9 \%$, el docente considera que es posible desarrollar conocimiento, presentando estrategias de aprendizaje, que le impliquen al estudiante, actividad significativa permanente, tanto en los cognoscitivo como en lo operativo.

El aspecto cognitivo es considerado por los docentes como un elemento importante en el desarrollo de las competencias; sin embargo, en sus propuestas de estrategias didácticas están ausentes las técnicas que posibiliten el desarrollo del pensamiento; la didáctica se caracteriza por tener un gran componente operativo - instrumental.

Lo operativo - instrumental se evidencia en el tipo de actividad propuesta para el desarrollo de las clases y la importancia de las estrategias; en la primera, la mayor de las frecuencias, están en actividades de tipo pasivo; en la segunda, el grado de importancia de las estrategias, el docente se lo concede, de manera contradictoria, a los procesos que generan altos niveles de pensamiento: la síntesis, el análisis, la comparación, la inducción, la deducción; todas aquellas que posibilitan el ascenso hacia formas de conocimiento más complejas.

Alcanzar los niveles cognitivos mencionados, solamente es posible si la estrategia didáctica planteada, brinda las condiciones para que el estudiante asuma un rol activo: retos cognitivos, situaciones complejas que requieren de procesos de análisis, de síntesis, entre otros; no así, desde un papel, como simple receptor de información, característico de la didáctica tradicional.

\section{Propuesta}

En consonancia con los resultados del marco del presente proyecto de investigación, se aporta al SENA y a las instituciones de formación profesional y técnica una propuesta para la construcción de un marco epistemológico, que fundamente los procesos de enseñanza-aprendizaje desde el modelo constructivista, para el desarrollo de competencias.

Esta propuesta se aborda desde dos referentes: el modelo pedagógico constructivista y el desarrollo de competencias: interrelacionados de manera sistémica generan un proceso dinámico, consistente, que fortalece la formación; por lo tanto, contribuye a mejorar la calidad educativa de las instituciones y sus efectos se reflejan directamente en el proyecto de vida personal, en el entorno social y laboral del país.

\section{El referente pedagógico: la investigación y la pedagogía}

Es prioritario crear un equipo pedagógico nacional cuyo principal propósito sea el de liderar el pro- 
yecto formativo institucional, desde un proceso de investigación educativa; su conformación debe ser abierta a todos los funcionarios interesados en participar, a partir de la consideración de unos perfiles determinados. Se sugiere tener en cuenta que un mayor número de integrantes sean pedagogos, educadores (docentes, formadores de docentes, investigadores), complementado con especialistas técnicos (según programas de formación) y funcionarios administrativos de alto nivel, con capacidad para la toma de decisiones.

Bien lo planteaba, La Misión Ciencia, Educación y Desarrollo, Colombia al Filo de la Oportunidad, (1997, p. 171), cuando al respecto afirmó:

\footnotetext{
Sin la investigación, la educación se convierte rápidamente en la transmisión mecánica y estática de información, negando así la posibilidad de desarrollar una capacidad de análisis y de comprensión, y una actitud innovadora que busque entender las relaciones existente ente los fenómenos biológicos, físicos y sociales.
}

La primera línea de investigación de la que se ocuparía este equipo es la pedagógica conformada por: el proceso de aprendizaje - enseñanza evaluación, el diseño curricular y la administración educativa institucional. Línea dada desde el más alto nivel hasta las coordinaciones académicas y técnicas de los centros de formación del país.

Algunos de los principales interrogantes que posiblemente puedan orientar el proceso investigativo de este equipo:

- ¿Qué tipo de ser humano está formando el SENA? ¿Para qué? ¿Por qué?

- ¿El tipo de ser humano que está formado el SENA es coherente con el entorno social, cultural, económico del país y responde a las necesidades de desarrollo en cada uno de estos aspectos?
- ¿El diseño curricular (programas de formación, guías de aprendizaje) y su desarrollo (estrategias didácticas, él énfasis en la formación por proyectos, la virtualización) garantizan la calidad formativa del estudiante del SENA? ¿Asegura el desarrollo de altos niveles cognitivos, actitudes éticas y destrezas operativas que articulen con un modelo de sociedad y de país?

- ¿El modelo pedagógico constructivista se ha hecho realidad, de manera integral, en las aulas de clase del SENA? ¿Es importante que así sea? ¿Por qué?

- ¿Qué tipo de relaciones se generan entre teoría y práctica? ¿Éstas son la fuente que retroalimenta el proceso de aprendizaje enseñanza y su consecuencia natural es el desarrollo de competencias?

- ¿La prevalencia de procesos diferenciados, heterogéneos de aprendizaje sobre la enseñanza homogenizadora es determinante en el desarrollo de las competencias?

- ¿Cómo posibilitar un proceso de aprendizaje desde los intereses, los ritmos, los estilos y las potencialidades de cada estudiante? ¿Él posibilitar dicho proceso es pertinente con el desarrollo de las competencias?

- LLa didáctica aplicada por el docente está en consonancia con el tipo de estudiante que determina el modelo pedagógico constructivista, adoptado por la entidad como base para el desarrollo de sus procesos de aprendizaje -enseñanza y el desarrollo de competencias?

- ¿Quién es el docente actual en el SENA? ¿Comprende y se apropia de su rol, para posibilitar la construcción de conocimiento y el desarrollo de competencias en el estudiante?

- ¿El actual sistema de gestión de calidad del SENA, aplica a los procesos de aprendizaje - enseñanza - evaluación, garantizado efectivamente el desarrollo de competencias? 


\section{La formación de los docentes}

Además de la investigación, la formación de los docentes se constituye en uno de los componentes principales del proyecto formativo institucional; éste adquiere gran relevancia, si se considera que es en el docente en quien recae la responsabilidad de liderar el enfoque de competencias y la gestión curricular que lo sustenta; es a él, a quien se le endilga el éxito o el fracaso de la política educativa institucional.

Tal compromiso debe ser asumido por profesionales docentes-investigadores, como única vía posible para abordar la problemática educativa institucional, definir fundamentos epistemológicos, antropológicos, sociológicos, psicológicos, pedagógicos de la formación profesional integral; para formular propuestas sobre el modelo pedagógico, sobre el desarrollo de competencias, sobre un diseño curricular coherente con los fundamentos definidos, sobre el proceso de administración educativa requerido para gestionar tales propuestas.

Es fundamental que se integren los procesos investigativos a la docencia, no solamente como práctica formativa, sino como parte de la programación del docente, con el fin de conformar una comunidad institucional de investigadores pedagógicos.

Es posible que las líneas de investigación descritas en esta propuesta sean susceptibles de ser abordadas desde el escenario de la formación de los docentes; gran parte de los interrogantes planteados han sido tratados en desarrollo del presente proyecto de investigación. La formación de los docentes es el escenario ideal, en donde puede germinar un proyecto de investigación pedagógica institucional serio, sobre el cual, se edifique la política formativa del SENA.

Otro de los aspectos claves en la formación de los docentes es el relacionado con la necesidad de afianzar el dominio de lo teórico, dado que la acción del docente del SENA se centra en lo práctico. Este propósito se puede lograr a través de estrategias didácticas activas que apunten al desarrollo de altos niveles cognitivos, cumpliendo con dos requisitos: el primero, que sean aplicadas en el proceso formativo del docente; el segundo, que el docente las aplique con sus estudiantes; al asegurar la relación permanente de teoría-práctica se motiva al docente para la realización de prácticas reflexivas, con sentido cognitivo.

Para que este tipo de aplicaciones prácticas se den en la realidad educativa institucional, se deben generar las condiciones para posibilitarla en términos de recursos, de programación, de revisar los procedimientos establecidos por la entidad y estandarizados por el sistema de calidad. Son estos mecanismos los que permiten trascender lo teórico en la formación del docente, para convertirla en una acción real en el aula.

\section{El enfoque de competencias y el proceso formativo}

La segunda línea de investigación en la que se ocuparía el equipo mencionado es la relacionada con el Enfoque de Competencias adoptado por el SENA; algunos de los siguientes interrogantes pueden servir como referentes:

- ¿Qué entiende el SENA por competencia y por competencia laboral? ¿Las divergencias y convergencias entre los dos conceptos son fortalezas susceptibles de reconstruirse?

- ¿Cuál es el propósito clave de la propuesta formativa del SENA al adoptar el enfoque para el desarrollo de competencias? ¿Este propósito está en consonancia con la misión y filosofía institucional de formación profesional integral?

- ¿La propuesta formativa de desarrollo de competencias, realizada por el SENA, ha impactado en la sociedad colombiana y en el sector productivo del país? ¿De qué forma? 
- ¿El modelo pedagógico constructivista aplica para el desarrollo de las competencias? ¿Se hace necesario rediseñar el enfoque institucional de éstas y su desarrollo formativo?

Dado que la base de la política educativa del SENA, se centra en el enfoque de competencias, es urgente crear una propuesta que resignifique la gestión formativa institucional, como lo plantea Barnett (2001, p. 249):

Lo que está en juego es una educación para el mundo de la vida humana. El mundo de la vida es más amplio que el de la competencia corporativa o el de la competencia académica, y las objeciones provendrán de los dos campos: ¿Cómo se puede justificar una educación superior para la vida? .... ¿Cómo ayudará este tipo de educación a generar una economía capaz de soportar el desafío de las naciones del Pacífico?

\section{El enfoque de competencias y el sector productivo}

El Manual de Diseño Curricular para el Desarrollo de Competencias en la Formación Profesional Integral (SENA, 2005, p. 70) plantea:

\footnotetext{
La educación es para el país el principal factor de competitividad y las exigencias que se presentan al sector productivo le generan nuevas y mayores demandas al sector educativo, para que el nuevo talento humano comprenda, apropie y aplique conocimiento, desarrolle habilidades, actitudes y valores requeridos para las especiales condiciones propias de nuestro proceso de desarrollo económico y social y las realidades del mercado internacional.
}

El desarrollo económico del país depende en gran medida de la formación de personas, que posean las competencias necesarias para su desempeño productivo, lo cual implica:
- El desarrollo de competencias cognitivas que les posibiliten la toma acertada de decisiones, el resolver situaciones problémicas complejas y el generar nuevos conocimientos para la construcción de proyectos de vida y de trabajo.

- El desarrollo de competencias básicas: asumir comportamientos éticos en la relación consigo mismo, con el otro y con el entorno. Parte integrante del entorno en donde se manifiestan los comportamientos éticos, lo constituye el mundo del trabajo.

- El desarrollo de competencias transversales para incorporar el desarrollo tecnológico en el fortalecimiento de los procesos investigativos y productivos del país.

En función de lograr el propósito de articulación entre el sector productivo y la formación profesional integral en el enfoque de competencias, el SENA debe realizar acciones más allá de las mesas sectoriales y del diseño de la norma de competencia.

Un mecanismo que posibilita el logro de este propósito es el de las evaluaciones de desempeño de los estudiantes en etapa productiva; éstas son el contacto directo con el empresario y facilitan la sistematización de su concepto sobre la formación recibida. Si se hace uso riguroso de este mecanismo, el SENA contaría con un insumo valioso para una evaluación de impacto, que le permita considerar la pertinencia y la calidad de la formación profesional integral en el enfoque de competencias.

A la información proporcionada por el empresario, tanto en las mesas sectoriales como en los procesos de evaluación institucional, se le debe dar un tratamiento académico. Limitar el análisis sobre la formación y la competencia a la óptica estrictamente productiva, contribuye a la polarización, ya existente en el SENA, entre proyecto formativo académico (competencia académica) 
o proyecto formativo operacional (competencia laboral), cuando en realidad no son excluyentes, sino complementarios.

Al respecto afirma Barnett (2001, p. 239):

Las dos definiciones a las que hemos llamado operacional y académica, contienen conjuntos alternativos de ideas relacionadas, que llevan a diferentes interpretaciones de la transferibilidad, la habilidad, la comunicación, las situaciones, el foco, la orientación, la crítica y la epistemología. Cada definición es coherente en sí misma, pero, al oponerse a la otra definición, resulta inevitablemente estrecha. Tenemos aquí opuestos polarizados -aun cuando el hielo se esté derritiendo y las aguas se estén mezclando-.

\section{Conclusiones}

Esta investigación posibilitó la identificación de las contradicciones entre el planteamiento teórico del Enfoque de Competencias del SENA, el modelo pedagógico constructivista que sustenta su desarrollo y la acción real del docente en el aula de clase.

EI SENA plantea la necesidad de fundamentar los actos pedagógicos en el desarrollo del pensamiento creativo, para ello, el docente debe privilegiar en sus acciones, los procesos más que los contenidos, según se establece en el Manual de Diseño Curricular para el Desarrollo de Competencias en la Formación Profesional Integral (20005); en este modelo los procesos de pensamiento son fundamentales para el conocimiento; estos se construyen de manera personal, al interior de la estructura cognitiva de la persona, mediante actividades didácticas significativas permanentes, caracterizadas por el rol activo del estudiante en su proceso de aprendizaje.

La forma como el docente ejecuta sus actos pedagógicos se ciñe de una parte, a los parámetros establecidos por El Procedimiento para la Ejecu- ción de Acciones de Formación Profesional Integral (2006) y controladas por el Sistema de Gestión de Calidad; de otra, a la actual exigencia directiva de presentar resultados concretos que del proceso formativo apliquen al sector productivo.

Estas dos líneas mencionadas, son contradictorias en sí mismas, pues mientras la primera (Manual de Diseño Curricular), propende por procesos de pensamiento; la segunda, insiste en resultados concretos y la estandarización del proceso de enseñanza-aprendizaje; asfixiando de esta forma, la propuesta formativa expresada en el marco teórico del Manual mencionado y contribuyendo grandemente a su extinción en la práctica real del docente.

El enfoque adoptado por el SENA, identifica dos características para las competencias laborales, la primera consiste en que, deben ser satisfactorias y aplicables a todas las organizaciones productivas del área objeto de análisis, la segunda, que se infiere de ellas, las capacidades relevantes y significativas que se plantearán como punto de partida para construir respuestas de formación profesional pertinentes, flexibles y de calidad (SENA, 1991); es inherente a este enfoque: la acción, la experiencia práctica, en coherencia con la filosofía institucional, fundada sobre la formación técnica para el trabajo.

La experiencia práctica se consolida, como el eje central del proceso de enseñanza-aprendizaje; éste último, solamente tiene validez en la medida que desarrolle las destrezas y habilidades que apliquen a las funciones propias del ámbito productivo.

Como efecto de lo anterior, los docentes privilegian en sus estrategias didácticas el hacer sobre el pensar, desconocen el valor de lo cognitivo como constitutivo esencial en el desarrollo de la competencia. Lo cual se evidencia en la realización de actividades de carácter pasivo: resumen de lectura; exposición del estudiante; textos escritos; toma de apuntes sobre un tema; desarrollo riguroso de la guía institucional; exposición del docente. 
Las estrategias didácticas activas son las que el docente aplica con menor frecuencia, desconoce las características fundamentales de éstas, especialmente la relacionada con el papel que cumplen en la generación de altos niveles cognitivos, en el fortalecimiento de valores y del proceso comunicativo; relega el papel activo del estudiante en su proceso de enseñanza-aprendizaje.

Es improbable, que con la poca frecuencia de aplicación de este tipo de estrategias, por parte del Docente, la cual oscila entre el $29.8 \%$ y el $21.25 \%$, logre que su estudiante desarrolle altos niveles cognitivos y fortalezca sus potencialidades de comunicación y valores, base sobre la cual se erige el desarrollo de las competencias.

Al limitar el desarrollo de las competencias básicas se evidencia la subordinación del concepto de competencia al concepto de competencia laboral; se pretende que el estudiante resuelva problemas concretos, de carácter práctico; una vez se presenta la solución, los docentes consideran desarrollada la competencia; es decir, asumen el concepto de competencia desde la acción, por la acción y para el desempeño.

Este modelo mental de los docentes es reforzado por la postura directiva institucional, que desde el discurso, menosprecia el valor de lo teórico, e insiste en lo procedimental, en los resultados, en lo concreto, como única posibilidad para lograr el desarrollo de las competencias y con ellas, apuntar a la pertinencia con el sector productivo del país.

El desarrollo curricular total del módulo o de la estructura curricular se afecta, como consecuencia de la reducida concepción del concepto de competencia y del proceso que sustenta su desarrollo; las temáticas se parcelan según la problemática planteada y con ellas, la visión de totalidad, de complejidad propia de la realidad, condición necesaria en el desarrollo cognitivo del estudiante; conocer es representar la realidad y generar la capacidad para intervenir en ella, es decir, ser competente para el mundo de la vida como totalidad, no para una de sus partes constitutivas, como lo es el mundo del trabajo.

Es urgente y necesaria la reflexión institucional, en torno a un marco epistemológico que dé claridad y precisión al enfoque de competencias adoptado por el SENA, en función de lo cual, es tarea prioritaria analizar los conceptos de competencia y competencia laboral, sus elementos constitutivos, el modelo pedagógico base del proceso de enseñanza-aprendizaje, y sobre el cual se sustenta el desarrollo de las competencias; la revisión del concepto de pertinencia, entre formación y sector productivo. Este último es el que debería plasmar la ineludible convergencia entre los intereses de la sociedad y los intereses del proceso formativo.

Contar con un marco epistemológico para el proceso de enseñanza-aprendizaje, en función del desarrollo de competencias (cognitivo, axiológico, comunicativo, procedimental, transversal), confiere significado y propósito a la acción formativa del SENA.

Las respuestas rápidas de carácter operacional y cuantitativo (cobertura) son insuficientes, no basta, con las respuestas tecnológicas (virtualización). Es preciso generar procesos de conocimientos que las sustenten, que transciendan el escenario educativo institucional y las meras necesidades productivas, para convertirse en riqueza intelectual en lo personal y en lo colectivo.

En cumplimiento de su misión y filosofía institucional, al SENA le corresponde dar una mirada pedagógica a su gestión. La formación profesional integral, en el enfoque de competencias, es compleja y requiere la reorientación del componente formativo que la connota. El aspecto laboral de la misma ha sido fortalecido en gran medida. Es el momento de considerar que la dinámica formativa institucional se constituye por la interrelación de ambos componentes; no así, por su consideración por separado, y con grandes dosis de antagonismo. 


\section{Referencias}

Argudín, Y. (2005). Educación Basada en Competencias. México: Trillas.

Argüelles, A. Compilador. (1996). Competencia Laboral y Educación Basada en Normas de Competencia. El Punto de Vista Pedagógico. México. Limusa Editores.

Barnett, R. (2001). Los Límites de la Competencia. Barcelona: Gedisa.

Bustamante, G. (2003). El Concepto de Competencia. Bogotá. Alejandría.

Casanova, F. (2002). Cambios en la Organización y Gestión de la Formación Profesional en América Latina y el Caribe. Brasil: CINTERFOR - OIT.

Varios. (2002). Reconocimiento y Certificación de Competencias Laborales: El Papel de los Sistemas de Formación. Brasil: CINTEFOR - OIT.

Varios. (2000). Competencias y Proyecto Pedagógico. Bogotá: Universidad Nacional de Colombia- Editorial Unibiblos.

Consejo de Normalización y Certificación de Competencia Laboral CONOCER. (1997). Sistema Normalizado y de Certificación de Competencia Laboral. México; CONOCER.

Gallego, R. (1999). Competencias Cognoscitivas. Un Enfoque Epistemológico, Pedagógico y Didáctico. Bogotá: Cooperativa Editorial del Magisterio.

García Márquez, G. (1997). La Proclama: Por un País al Alcance de los Niños. En: Colombia al Filo de la Oportunidad. Bogotá: Instituto para La Investigación Educativa y el Desarrollo Pedagógico - IDEP.
Lucio, R. (1978). 20 Años del SENA en Colombia 1957 - 1977. Bogotá. Fundación para la Educación Superior y el Desarrollo - Editorial Presencia.

Mertens, Leonard. (1996). Competencia Laboral. Sistemas, Surgimiento y Modelos. Montevideo: CINTERFOR - OIT.

Misas, G. y Equipo de Consultores, (1994). El SENA del Siglo XXI. Elementos para su Reestructuración. Tomo 1, 12 y 3. Bogotá: SENA.

Moreira, M. (2000). Aprendizaje Significativo: Teoría y Práctica. Madrid: Visor.

Morin, E. (1999). Los Siete Saberes Necesarios para la Educación del Futuro. Bogotá: UNESCO - Ministerio de Educación de Colombia.

Gómez Buendía, H. (1999). Educación. La Agenda del Siglo XXI. Programa de Naciones Unidas para el Desarrollo. Bogotá: TM Editores.

POZO, J. (1994). Teorías Cognitivas del Aprendizaje. Madrid. Ediciones Morata.

SÁNCHEZ, M. (2002). SENA 45 Años. Bogotá. Imprenta Nacional

SENA. (1999). Competencias Laborales: Base para Mejorar la Empleabilidad de las Personas. Bogotá. SENA - Dirección General

SENA - Dirección General - CORPOEDUCACIÓN. (2001). El Estado del Arte de las Competencias Básicas o Esenciales. Bogotá. SENA-Dirección General.

SENA- Dirección General. (2005). Guía Metodológica para la Administración Educativa de la Formación Profesional Integral del SENA. Bogotá. SENA - Dirección General.

SENA - Dirección General. (1986). Estrategias Didácticas para la Formación Profesional. Bogotá. Subdirección Técnico - Pedagógica. 
SENA - Dirección General. (1997). Estatuto de la Formación Profesional Integral del SENA. Bogotá. SENA - Dirección General.

SENA- Dirección General. (2005). Manual para Diseñar Estructuras Curriculares y Módulos de Formación para el Desarrollo de Competencias en la Formación Profesional Integral. Bogotá. Dirección de Formación Profesional, División de Investigación y Desarrollo Técnico-Pedagógico.

SENA- Dirección General. (2003). Metodología para Evaluar y Certificar Competencias Laborales. Bogotá. SENA- Dirección General.

SENA - Dirección General. (2001). Manual de Evaluación y Certificación con Base en Normas de Competencia Laboral. Bogotá. SENA - Dirección General
SENA - Dirección General. (1986). Unidad Técnica. Tecnología Educativa. Bogotá. SENA - Dirección General

SENA, Dirección de Empleo, División de Estudios Ocupacionales. (1999). Guía para la Elaboración de Normas de Competencia y Titulaciones, con base en el Análisis Funcional. Bogotá. SENA - Dirección General

SENA, Dirección General, Dirección del Sistema Nacional de Formación Profesional. (2001). Sistema Nacional de Formación para el Trabajo Modelo Colombiano. Bogotá. SENA Dirección General

SENA - Regional Distrito Capital. (2007). Proceso de Integración de Competencias. Las Competencias Básicas. Bogotá: SENA.

SKINNER, B. F. (1986). Sobre el Conductismo. Barcelona: Orbis. 